Journal of Engineering and Applied Sciences 14 (Special Issue 3): 6019-6025, 2019

ISSN: 1816-949X

(C) Medwell Journals, 2019

\title{
Surface Profile Determination by using Developed Profilometer
}

\author{
${ }^{1}$ Samaa Mahdi Salih, ${ }^{2}$ Emad Kamil Hussein and ${ }^{1}$ Kadhum Ahmed Abed \\ ${ }^{1}$ Department of Mechanical Engineering, Al-Anbar University, University of Anbar, Ramadi, Iraq \\ ${ }^{2}$ Department of Pumps Engineering, Al-Furat Al-Awsat Technical University, Kufa, Iraq \\ Samaaalqaysi88@gmail.com,kadhum1968@yahoo.com, emad_kamil72@tcm.edu.iq
}

\begin{abstract}
A developed profilometer was designed and manufactured to determine different mechanical profiles depending on tactile sensing technique. The basic concept of the profilometer work depends on direct contact between the stylus tip and the measurand part. The design is robust and suitable to measure the selected part. The surface profile of a cam shape was measured by using this profilometer. The obtained profile has a big match with the actual dimensions of the measurand part. Cross correlation function also was studied to find the best matching between profiles.
\end{abstract}

Key words: Tactile sensing, profilometer, Surface profile measurement, correlation function, measurand part, big match

\section{INTRODUCTION}

Tactile sensing covers all methods in which a sensor comes in a physical contact with the measured object. many variables can be measured by contact like position, temperature, pressure and shape (Lederman and Klatzky, 1987). In some critical applications, the dimensions and diameters cannot be measured with high precision and in traditional ways, so, the idea of design and manufacture a profilometer was generated. Measuring the surface profile (actual shape) of any mechanical part is the main purpose of this study. Measurement of a surface profile for any mechanical part is a vital area in many of today's industries, the importance of profile measurement appears in achieving the specification and also to ensure that any manufacturing process is in control and not producing excessive or inadequate profile height. Many researchers using tactile sensing to get the shape of the object and detection edge. By Canny (1986) was introduced a computational approach to edge detection where the researcher described a procedure for the design of edge detectors of arbitrary edge profiles, this design relied on the specification of detection and localization criteria in mathematical form. Numerical optimization was introduced to detect optimal operators for roof and ridge edges (Canny, 1986).

Some researchers have focused on the reconstruction of a full shape of unknown objects using tactile sensors by introduced an integrated approach, to robotic haptic object recognition that tries to feature based approaches and to blend 3D Model to object recognition with the capability to sense objects by both tactile feedback and kinaesthetic (Allen and Roberts, 1989). Others offered a method to reconstruct the motion and the shape of the unknown smooth convex object, the object was manipulated by planner palms covered with tactile elements. The motion and the shape of the object can be written as a function of the motion of the palms and the sensor values. The offered method used in an application in grasping unknown objects and tactile exploration of the unknown environment. The method showed that the 3D case was essentially different from the planner case (Moll and Erdmann, 2004).

Another researchers presented an approach for object identification using tactile sensors installed in the fingertips of a manipulation robot, this approach worked on low-resolution intensity images which were achieved when an object was grasped by the robot, a bag of words approach was applied by means of unsupervised clustering on data, this approach learned a vocabulary from tactile reading which was used to form a histogram codebook when the objects were larger than the sensor, the robot required multiple grasp actions at various position to identify an object to minimize the required grasp actions a decision theoretical framework was applied which reduce entropy of the probabilistic belief about the type of the object after that this approach was validated by experimental

Corresponding Author: Samaa Mahdi Salih, Department of Mechanical Engineering, Al-Anbar University, University of Anbar, Ramadi, Iraq, Samaaalqaysi88@gmail.com 
research that showed the ability of the approach to distinguish between a large set of objects (Schneider et al., 2009).

In 2010, two techniques for haptic object recognition were used which they were passive joints and haptic key features an approach for tactile object recognition with an anthropomorphic robot hand primary, passive degrees of freedom was presented to tactile sensor system of the robot hand which permitted the planar tactile sensor spots to optimally adjust themselves to the surface of the object and to obtain extra sensor information for shape rebuild then an approach was presented to categorize an object directly by using tactile sensor data that obtained by the palpation sequence with the robot hand without structure a $3 \mathrm{D}$ Model of the object a limited set of palpable contact patterns and vital finger positions were recognized that can be used to describe a single tactile step, a palpation system can then be combined in to a simple numerical description of the object and then classified (Gorges et al., 2010).

Another tactile sensor system was introduced with high spatial and force resolution which gave the ability to get robust recognition and $6 \mathrm{D}$ localization of static object and also free standing movable objects in deep-sea environments, the researchers can robustly evaluate highly informative local surface features of an objects by using locally dense point clouds collected from touch, so, feature matching algorithm used for this purpose by using tactile sensors (Aggarwal and Kampmann, 2012). Some researchers used a stylus type surface profilometer and analyzed the $2 \mathrm{D}$ and $3 \mathrm{D}$ distortion effects of the measured profile which were caused by the stylus tip radius. Stylus tip selection criteria were suggested to obtain a suitable tip radius depends on surface texture characteristics of the specimen.

This criterion for improving the reliability of measurement results. The developed simulation model made possible the creation of different stylus tip shapes but only the results obtained by using spherical tip radius was proposed to simplify the tracing error calculation and compare the measurement results with simulation results. The 3D surface texture used in the simulation was obtained using an atomic force microscope from an actual machined surface. The simulation results were compared with the measured results from the roughness tester for the same specimen. Cumulative power spectral analysis was performed for the $2 \mathrm{D}$ and $3 \mathrm{D}$ simulated profiles to examine the distortion of the wavelength caused by tip radius which appears shorter than the point where the cumulative power becomes $95 \%$ of the total power regarded to be effective (Lee and Cho, 2012).

In 2016 a robot manipulator was developed which used in industrial welding process, the system with the tactile sensor used to execute various tasks in order to recognize position, shape and orientation. This type of system had the ability to pick and place any object which defined previously from a conveyor system. The control of the robot was done by DC motor based sensor values. Scanning of object edges was possible with $3 \mathrm{D}$ surface relied on the angle change. A number of sensors were put in a conveyor and in the robot manipulator when the values of the sensor in each conveyor and the robot were larger than the recognized position edge then the object could not be lifted from the conveyor system. When the angle of the sensor in the conveyor system was smaller than the edge of the robotic hand then the object was lifted from the conveyor system. The object recognition and lifting relied on the position of the robotic arm and edge scanning (Hussain et al., 2016).

\section{MATERIALS AND METHODS}

Design and implementation: Tactile displacement sensor that used in this study is composed from the following mechanical parts: barrel cylinder made of wrought aluminum alloy with $33 \mathrm{~mm}$ diameter with a square cross sectional area $\left(43 \times 43 \mathrm{~mm}^{2}\right)$ cover made from aluminum die cast coated, $202 \mathrm{~mm}$ length. The central piston has a diameter of $32 \mathrm{~mm}$, piston rod from high alloy steel has a length of $290 \mathrm{~mm}$ and $12 \mathrm{~mm}$ diameter. While the stylus is made from stainless steel with $73 \mathrm{~mm}$ length $3 \mathrm{~mm}$ diameter and the probe is hard, smooth with an aspect ratio between the stylus diameter and its length of 0.054 and has a rounded tip with a small diameter of $4 \mathrm{~mm}$ that can detect the measured surface profile easily.

The flat ends spring has a free length of $120.25 \mathrm{~mm}$ inner diameter $27.5 \mathrm{~mm}$ outer diameter, the coil diameter of the spring is $2.5 \mathrm{~mm}$. The required reading from this sensor was obtained by using an electrical rheostat which applied on the top of the sensor and still in touch with the movement of spring. Then this rheostat was connected to a digital multi meter to take the resistance values with each movement of the sensor. Figure 1 demonstrated the designed three dimensional tactile sensor and Fig. 2 elucidated the developed tactile sensor. 


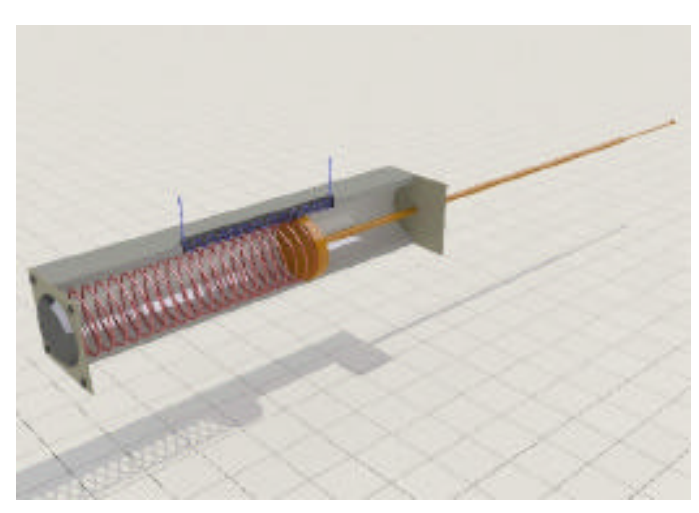

Fig. 1: Internal design of the tactile sensor

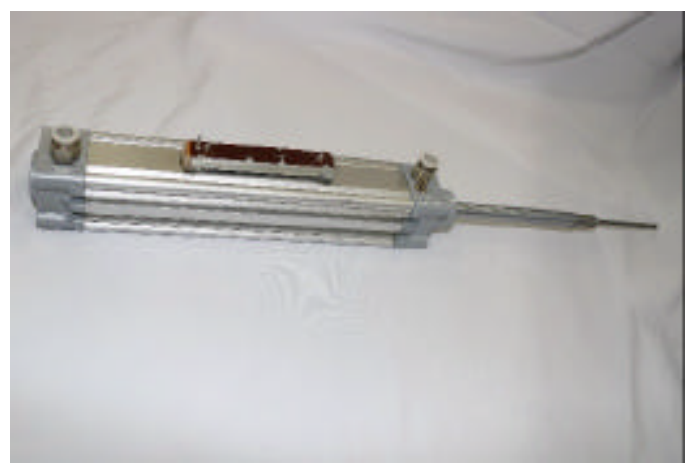

Fig. 2: The developed tactile sensor

Starting design implementation with making a slot by using a drill tool size $5 \mathrm{~mm}$ in the middle of the case from the top with small dimensions about $85 \mathrm{~mm}$ length and $5 \mathrm{~mm}$ wide that allow to employ the electrical rheostat on it and ensure that the movement of the electrical rheostat is very smooth through this small slot. Fixing the electrical rheostat in a way that can be changed easily. The stylus has a length of $73 \mathrm{~mm}$ and fixed inside the piston rod after made a suitable small hole by using a drilling tool size $4 \mathrm{~mm}$ that fit with stylus dimensions. A spring which its properties were mentioned previously was employed behind the central piston. The developed tactile displacement sensor (profilometer) work by direct touch between its stylus and the workpiece.

Relation between displacement and the electrical resistance: The slider rheostat or slider potentiometer are used in many applications like motion control, audio control, home electrical appliances. Like these rheostats are designed to have changed in their contact resistance values by the linear motion of its slider. So, there is a relationship between the output resistance and the slider movement. The resistance element of potentiometer has

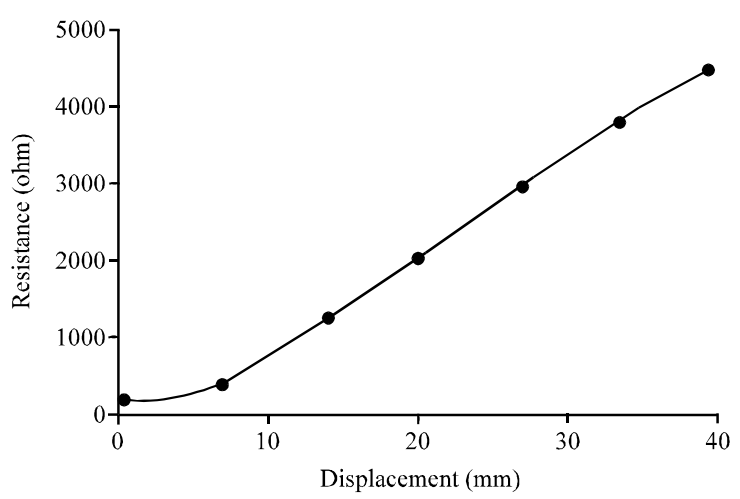

Fig. 3: The linear relationship between displacement and resistance

Table 1: Showed the relation between the displacement and its corresponding resistance

\begin{tabular}{lc}
\hline Displacement $(\mathrm{mm})$ & Resistance $(\Omega)$ \\
\hline 0 & 200 \\
5 & 440 \\
10 & 1310 \\
15 & 2140 \\
20 & 3100 \\
25 & 3950 \\
\hline
\end{tabular}

a linear characteristic or a linear change between the range of travel and the resistance during this travel range. To prove that connecting the rheostat with a multimeter and measure the amount of resistance with each movement of its slider during its whole track. The following results illustrated in Table 1 are obtained which showed the linear characteristic between the two features that illustrative in Fig. 3. The slope of Fig. 3 found to be 170 and the intersection point between the two axes was 200 then the linear equation between displacement and resistance was (Table 1):

$$
y=170 x+200
$$

which demonstrated that the resistance is a function of displacement. This equation will be used to convert the obtaining resistances from the digital multimeter to its corresponding displacement which represented the radius of the measurand part. The relationship between the physical measurement variable input and the signal variable output for a specific sensor is represented the sensitivity of the sensor and it is known as the calibration of the sensor.

Work procedure: The measurand part which was a cam shaft was fixed between the chunk and the tailstock of the lathe machine and the developed tactile displacement sensor was fixed in the cross slide of the lathe machine and keep it in contact with the measurand part by rotating 


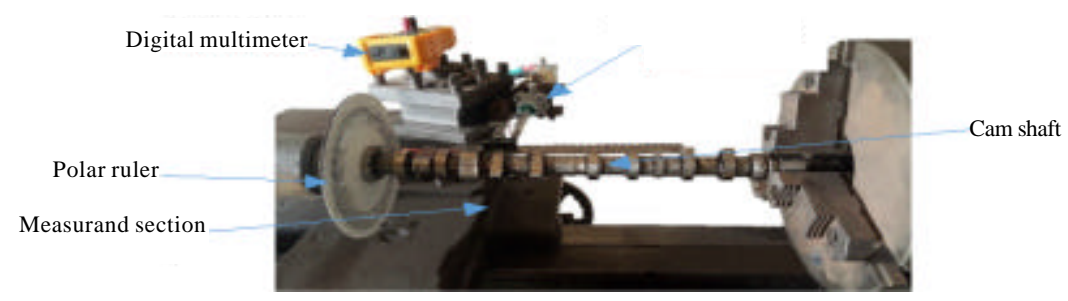

Fig. 4: Tactile sensor connecting method

the measurand part manually ten degrees each time of movement start from zero degree until reach $360^{\circ}$ by using a simple polar ruler to facilitate the manual movement of the measurand part. The tip sensor starts sense the rotating part with the movement of the sensor, the spring start in compressing and expanding this movement of spring causes rheostat movement. During rheostat movement the digital multimeter which was in contact with the rheostat from rheostat two terminals ends begins record resistance reading. These resistances reading were converted to its analogue value of displacements. These displacements represented the value of the measurand part radius, according to Eq. 1 and Fig. 4 demonstrated the work setup.

\section{RESULTS AND DISCUSSION}

After following the above measurement protocol to obtain the surface profile for the used measurand part. The obtained readings were plotted also by using STATISTICA Version 12. Figure 5-7 will demonstrate the cam profile in the polar coordinate, Cartesian coordinate and also the actual dimensions for the cam profile. The used of the spring in the current study maintain positive contact between the profilometer components. The stylus compliance and reduction in sensitivity is an essential limitation for the aspect ratio between the stylus radius and its length. Approximately an aspect ratio of 0.054 that used in probe design give a good accuracy in reading and increase the sensitivity of the used sensor. This ratio coincided with.

It's obvious from the obtained results that the design was robust where the measurand part has a big matching in measurement with the values of the known actual shape except in some places where some errors were founded. The maximum value of error in cam profile measurement found to be $1.87 \mathrm{~mm}$ while the minimum 1 was $0.11 \mathrm{~mm}$. These small errors related to the wear which happen in the used cam shaft because it was already used in order to compared the used cam shaft with the original one and find best matching between them a profile shifting process by using a cross correlation function as will be demonstrated in the next section. One of the most important functions which is used in analyzing phase matching between any two geometrical engineering profiles is the cross-correlation function which has a general formula as follows:

$$
\mathrm{F}(\mathrm{t}) \mathrm{f}_{1} \mathrm{f}_{2}=\frac{1}{\mathrm{~N}} \sum_{\mathrm{n}=0}^{\mathrm{N}-1} \mathrm{f}_{1}(\mathrm{n}) \mathrm{f}_{2}(\mathrm{n}+\mathrm{t})
$$

Where:

$\mathrm{F}(\mathrm{t})_{\mathrm{ff} 2}=$ Cross-correlation function

$\mathrm{N}=$ Number of samples

$\mathrm{f}_{1}(\mathrm{n})=$ First function value

$\mathrm{F}_{2}(\mathrm{n}+\mathrm{t})=$ Second function value

$\mathrm{t}=$ Shifting step

Figure 8a showed the results of applying the cross-correlation function on the cam shaft. It could be noticed that the maximum value of the function is equal to 389.12 at an angle of $30^{\circ}$. In order to get the best matching in cam profiles, the cam shaft should be rotated by $30^{\circ}$ counter clock-wise direction. To enable profile comparison an error measure was done to denote the deviation between the measured profiles and the actual one. This error is defined as follow:

$$
E(n)=\left|f_{1}(n)-f_{2}(n)\right|
$$

Where:

$\mathrm{E}(\mathrm{n})=$ Error function

$\mathrm{f}_{1}=$ First function value

$\mathrm{f}_{2}=$ Second function value

The error between the two cam profiles was computed by using the given profile orientation without shifting and its results as shown in Fig. 8b. Figure 8 shows in detail that the error which is represented by the area under the curve is of high value. The second case is calculating the error but after shifting the measured profile by $30^{\circ} \mathrm{CCW}$. Figure $8 \mathrm{c}$ showed the computed error by comparing those two cases. According to the results, the 


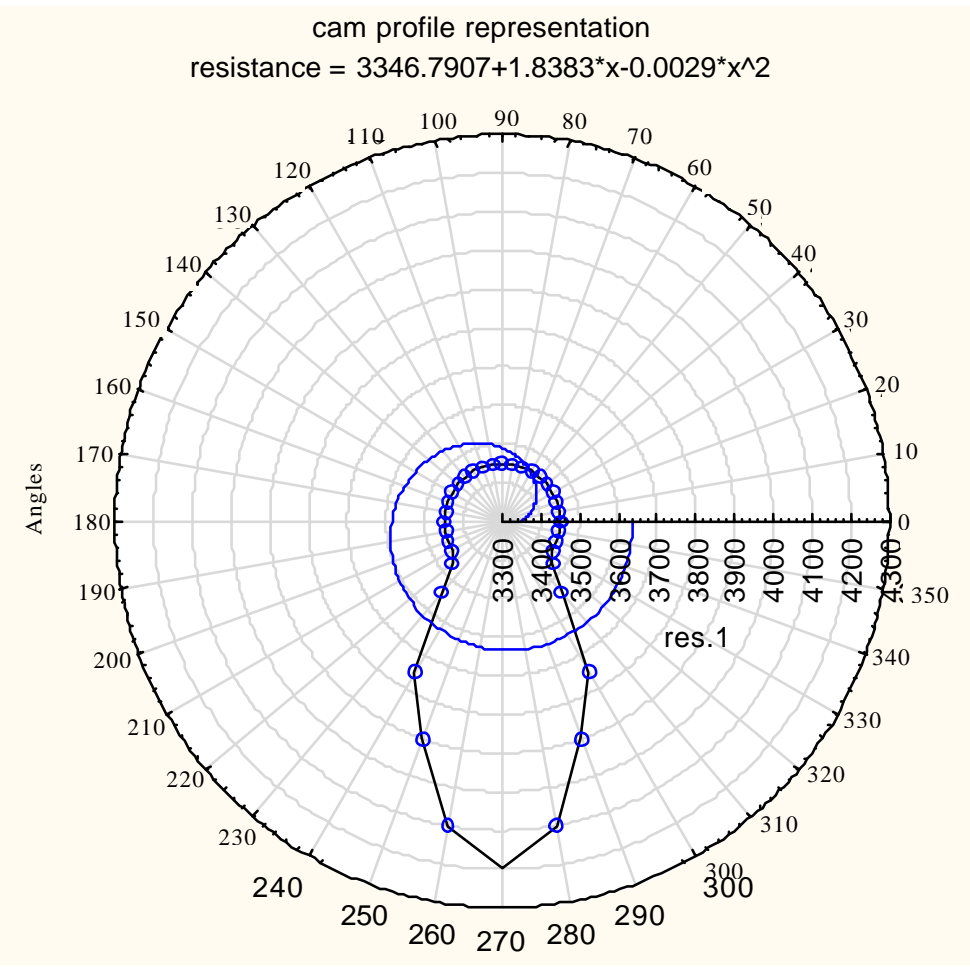

Fig. 5: Standard cam profile; Scatterplot $\mathrm{f}$ res. 1 against angles cam profile representation resistance = $3346.79 .7+1.8383^{*} \times-0.0029 * \times 2$

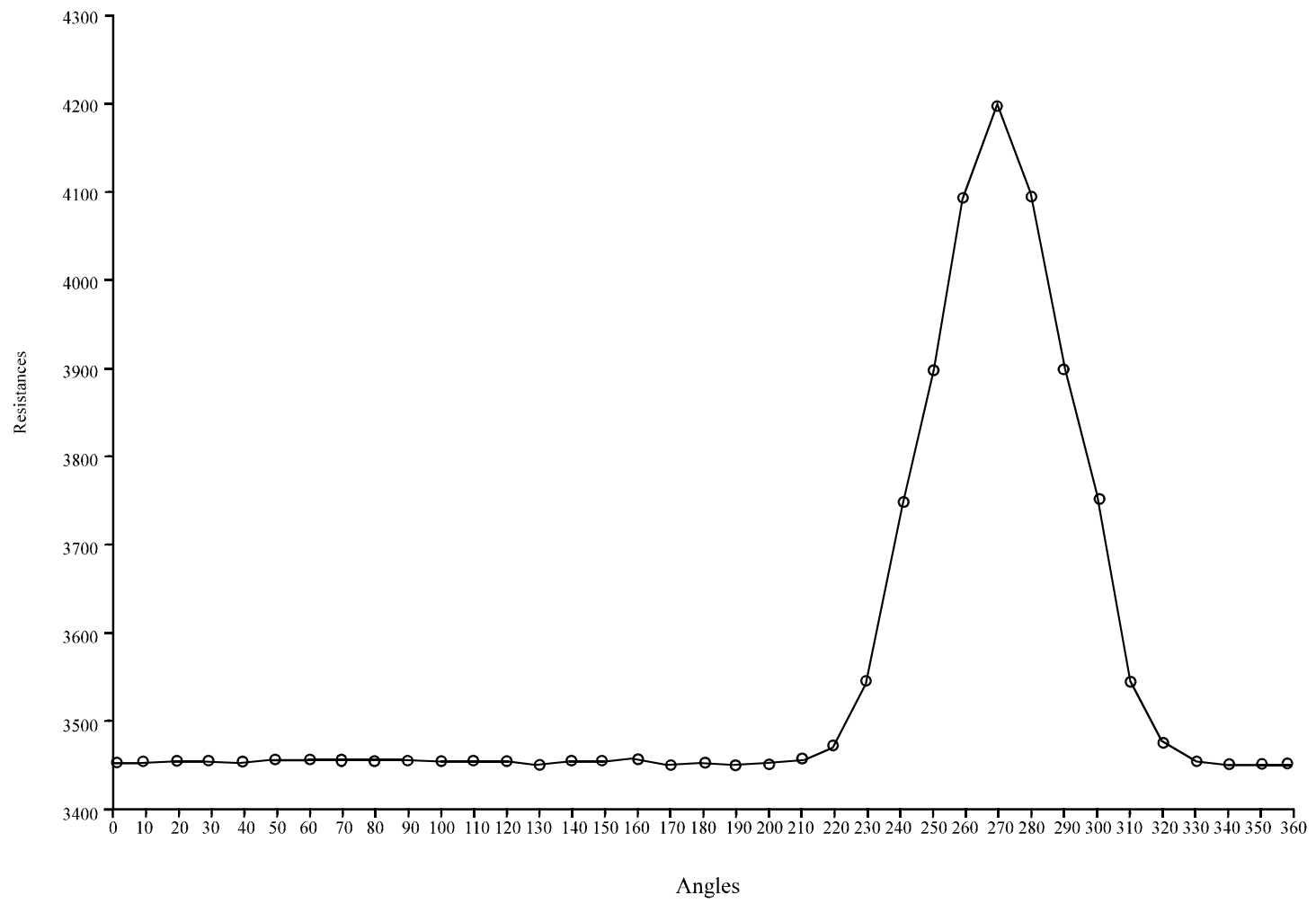

Fig. 6: Standard cam profile signal: Scatterplot $\mathrm{f}$ res. 1 against angles cam profile representation 


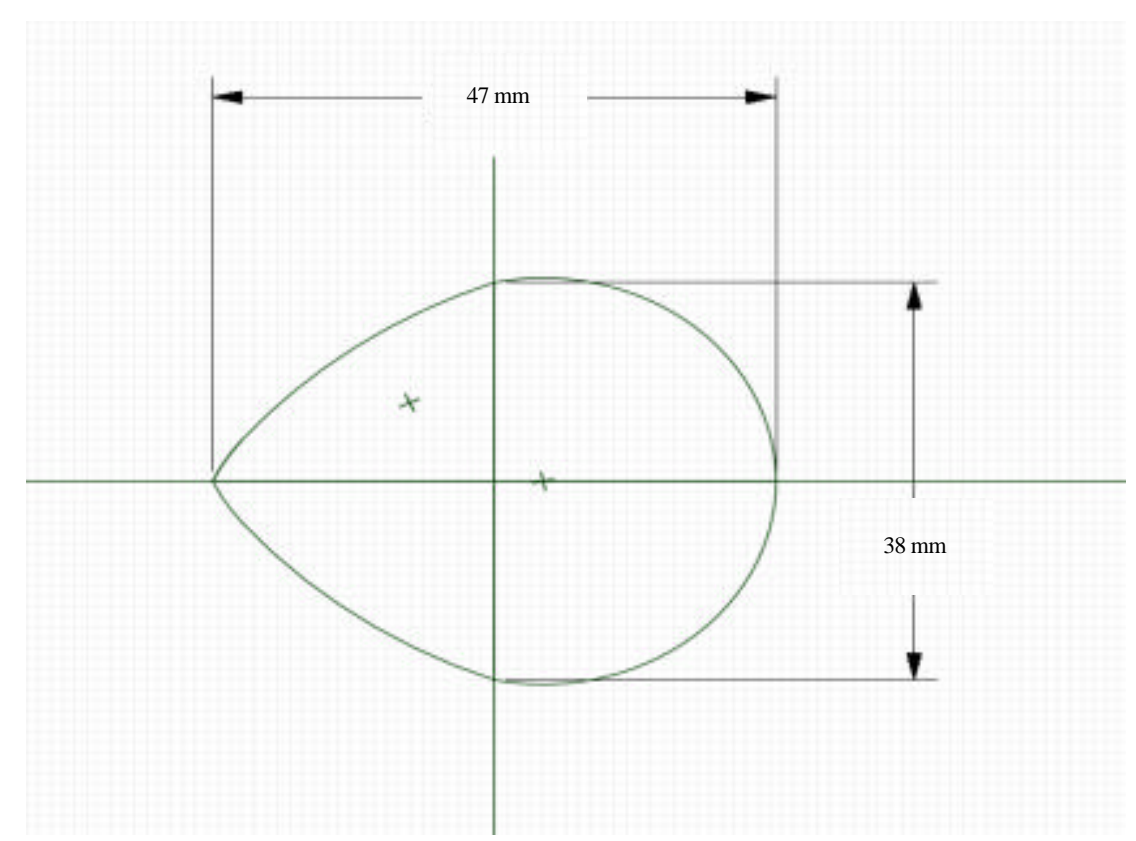

Fig. 7: Geometrical graph for the cam profile
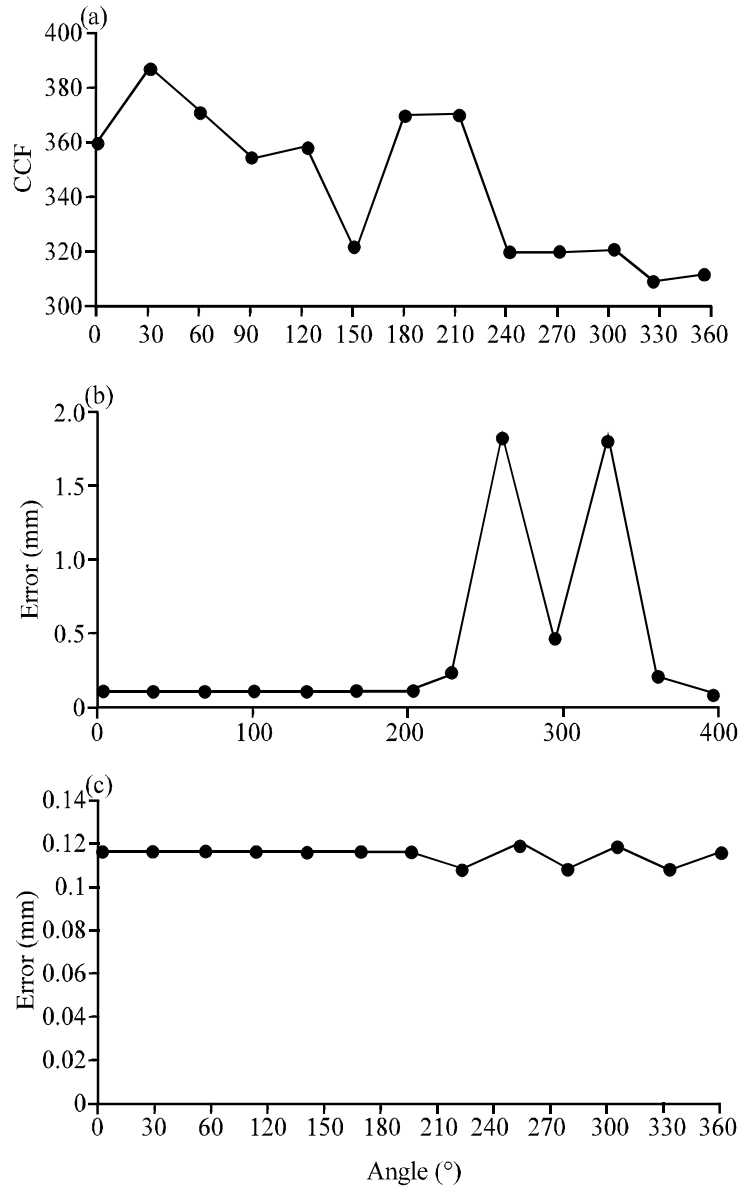

Fig. 8: a-c) The results of the cross correlation processing difference between the two error is very large. The error after shifting the measured second cam to its best matched orientation is much less, therefore, the matching after shifting is better than before shifting.

\section{CONCLUSION}

A developed profilometer was designed and manufactured according to the desired specifications. The cross-sectional profile of the measurand part (cam profile) was obtained by using the developed profilometer. The employed spring in the current study maintain positive contact between the profilometer components. The design was robust enough to measure different topography where the error found to be as small as possible. Another consideration should be taken into account is the simplicity of probe design. When the stylus system is a simple one this will maintain a better accuracy of the measurement. Always straight single stylus gives the better performance than another stylus with joints and bends. Probe length plays important role in reducing measurement errors. The long probe may cause bending, increase sensor errors such as pitch, yaw, straightness and twist. The short and lighter probe will give the measurement maximum sensitivity. After that applying the cross correlation function on the measured values it is obvious that the matching after shifting is better than before shifting and the errors were reduced to its minimum values. All contact parts and surfaces will suffer from local wear and erosion, so, it is impossible to use such worn tools longer. 


\section{REFERENCES}

Aggarwal, A. and P. Kampmann, 2012. Tactile sensors based object recognition and $6 \mathrm{D}$ pose estimation. Proceedings of the International Conference on Intelligent Robotics and Applications, October 3-5, 2012, Springer, Berlin, Heidelberg, Germany, ISBN:978-3-642-33502-0, pp: 406-416.

Allen, P.K. and K.S. Roberts, 1989. Haptic object recognition using a multi-fingered dextrous hand. Proceedings of the 1989 International Conference on Robotics and Automation, May 14-19, 1989, IEEE, Scottsdale, Arizona, ISBN:0-8186-1938-4, pp: 342-347.

Canny, J., 1986. A computational approach to edge detection. IEEE Trans. Pattern Anal. Mach. Intell., 8: 679-698.

Gorges, N., S.E. Navarro, D. Goger and H. Worn, 2010. Haptic object recognition using passive joints and haptic key features. Proceedings of the 2010 IEEE International Conference on Robotics and Automation, May 3-7, 2010, EEE, Anchorage, Alaska, ISBN:978-1-4244-5038-1, pp: 2349-2355.
Hussain, S.A., C. Ramaiah, M. Prabhu and S.J. Hussain, 2016. Robot manipulator with a tactile sensor for object recognition in industrial welding process. Intl. J. Control Theor. Appl., 9: 8373-8381.

Lederman, S.J. and R.L. Klatzky, 1987. Hand movements: A window into haptic object recognition. Cognit. Psychol., 19: 342-368.

Lee, D.H. and N.G. Cho, 2012. Assessment of surface profile data acquired by a stylus profilometer. Meas. Sci. Technol., 23: 1-12.

Moll, M. and M.A. Erdmann, 2004. Reconstructing the Shape and Motion of Unknown Objects with Active Tactile Sensors. In: Algorithmic Foundations of Robotics V, Boissonnat, J.D., J. Burdick, K. Goldberg and S. Hutchinson (Eds.). Springer, Berlin, Heidelberg, Germany, ISBN:978-3-642-07341-0, pp: 293-309.

Schneider, A., J. Sturm, C. Stachniss, M. Reisert and H. Burkhardt et al., 2009. Object identification with tactile sensors using bag-of-features. Proceedings of the 2009 IEEE/RSJ International Conference on Intelligent Robots and Systems Vol. 9, October 10-15, 2009, IEEE, St. Louis, Missouri, ISBN:978-1-4244-3803-7, pp: 243-248. 